\title{
TECNOLOGÍAS LOCALES DE PRODUCCIÓN DE ARRACACHA (Arracacia xanthorrhiza Bancroft) EN EL MUNICIPIO DE BOYACÁ, DEPARTAMENTO DE BOYACÁ
}

\section{LOCAL TECHNOLOGIES OF ARRACACHA (Arracacia xanthorrhiza Bancroft) PRODUCTION AT BOYACÁ, DEPARTMENT OF BOYACÁ}

\author{
Álvaro Alvarado Gaona¹, Lyda Ochoa²
}

\begin{abstract}
${ }^{1}$ Ingeniero Agrónomo, M.Sc. Desarrollo Rural. Profesor asistente, Facultad de Ciencias Agropecuarias, Universidad Pedagógica y Tecnológica de Colombia, Avenida Central del Norte, Tunja. E-mail: alvaro.alvarado@uptc.edu.co. ${ }^{2}$ Ingeniera Agrónoma., Joven Investigadora Grupo CREPIB, Universidad Pedagógica y Tecnológica de Colombia, Tunja. E-mail: lydaochoa95@yahoo.es.
\end{abstract}

Rev. U.D.C.A Act. E Div. Cient. 13 (1): 125-133, 2010

\section{RESUMEN}

La base de la economía del municipio de Boyacá es la producción agrícola y pecuaria. La arracacha es un producto de gran importancia agro-alimentaria y socio-económica que, alternada con la papa, se cultiva en parcelas de pequeños agricultores, quienes han adquirido tradicionalmente sus conocimientos sobre el sistema de producción. Debido a la demanda del mercado por ciertas variedades, el caso de paliverde y yema de huevo, como se denominan allí, se han dejado de cultivar otras, como la blanca de tarro y la amarilla de tarro, favoreciendo la pérdida de agro-biodiversidad. El objetivo del presente estudio fue describir los aspectos relevantes de este sistema de producción, a partir de la información recopilada sobre materiales cultivados, manejo agronómico y tecnologías locales de cultivo, partiendo del conocimiento acostumbrado de sus agricultores. Se siguió una metodología participativa, mediante la aplicación de encuestas. Los resultados indicaron que en Boyacá se encuentran más de siete materiales genéticos: paliverde, palirrusia, palinegra, yema de huevo, yucatana, blanca de tarro y amarilla de tarro (denominaciones locales). Se siembra en asociación con leguminosas y/o gramíneas o intercalada con frutales, como el tomate de árbol. La incidencia de plagas y de enfermedades es baja; una pudrición de raíz es la más relevante (Sclerotinia sclerotiorum). El rendimiento varío entre 2 y $4 \mathrm{~kg} /$ planta (peso total de la raíz). La arracacha es una especie con gran potencial por sus características nutricionales y usos agro-industriales, por tanto, se deben plantear estrategias, con el objeto de mejorar la rentabilidad del cultivo y conservar y mejorar los materiales genéticos existentes.

Palabras clave: Raíces andinas, manejo agronómico, sistema de producción agrícola, agro-biodiversidad.

\section{SUMMARY}

The economic support of Boyacá Municipality (state of Boyacá) is agriculture and livestock production. "Arracacha" (Arracacia xanthorrhiza) is a high value agro-alimentary and socioeconomic product. Its growing is alternately with potato in small fields by local farmers who have acquired their knowledge traditionally through generations. Due to the high market demand for some varieties like "paliverde" and "yema de huevo", as named in the region, farmers have stopped to seed other cultivars such as "blanca de tarro" and "amarilla de tarro", leading 
consequently to an agro-diversity loss. The objective of this study was to characterize this production system based on gathering information on cultivated varieties, agronomic management, local crop technologies, and farmers' traditional knowledge. A participatory method through survey collection, interviews and field visits was followed. The results showed that in Boyacá there are more than seven genetic materials: paliverde, palirrusia, palinegra, yema de huevo, yucatana, blanca de tarro and amarilla de tarro, as denominated locally. They are produced in association with leguminous and maize crops or with fruit trees as Cyphomandra betacea (tomate de árbol). Pest and disease incidence is low. Root rotting (Sclerotinia sclerotiorum) is the most notorious disease. The yield varies between $2-4 \mathrm{~kg} /$ plant (total root weight). Arracacha has high agro-industrial potentials due to its nutritional characteristics and agro-industrial uses. Therefore, research to improve crop yield must be considered and the existing genetic materials must be preserved and improved as well.

Key words: Andean roots, agronomic management, agricultural production system, agricultural biodiversity.

\section{INTRODUCCIÓN}

El cultivo y el aprovechamiento de la arracacha (Arracacia xanthorrhiza Bancroft), datan de la época precolombina. Los únicos indicios son restos de raíces encontradas en tumbas del Perú antiguo, sin indicarse la localización (Safford, 1917 citado por Seminario, 2004). Vásquez et al. (2004) refieren a Colombia como parte del centro de diversidad primaria del género Arracacia, la cual, se ha mantenido gracias a etnias y culturas indígenas que la cultivan para venta o auto-consumo. Hermann (1997) indica que la arracacha ocupa un lugar secundario en la dieta de cerca de 80 a 100 millones de personas en América del Sur.

La planta de arracacha produce varias raíces laterales, que constituyen la parte comestible, un rizoma central y varios tallos laterales cortos o renuevos, que sirven como propágalos, así como un exuberante follaje. Es una especie perenne, pero se cultiva como anual (Knudsen et al. 2006).

La arracacha se produce principalmente en Brasil, en Colombia, en Ecuador y en Venezuela, donde es un producto regular de los mercados urbanos (Hermann, 1997). En Perú, se conoce con los nombres de racacha y virraca (Seminario, 2004); en Ecuador, como zanahoria del país o zanahoria blanca; en Venezuela, como apio criollo (Jiménez, 2005) y racacha (Amaya E Julca, 2006) y en Brasil, mandioquinha-salsa (Heredia et al. 2009) y batata baroa (Amaya E Julca, 2006), entre otros. Knudsen et al. (2004) indican que a pesar que se ha llevado repetidamente a otras regiones, no se ha logrado establecer como cultivo comercial, con excepción de Brasil, donde se siembra más arracacha que en cualquier país andino.

El Ministerio de Agricultura y Desarrollo Rural - MADR reporta que en Colombia, para el 2008, se cultivaron un total de $6442 \mathrm{ha}$, siendo Tolima el principal productor (3235ha), seguido por Norte de Santander (683ha), Huila (563ha) y Boyacá (517ha). Según esta misma fuente, los rendimientos estuvieron entre 4,76t/ha, en Cauca y 17,4 t/ha, en Antioquia. Ese mismo año en el Departamento de Boyacá, el municipio de Tibaná fue el primer productor, con $2400 \mathrm{t}$, seguido por el municipio de Boyacá, con 1560t (MADR, 2008), siendo uno de los cultivos de mayor importancia en la economía agrícola, junto con la papa (POT Municipal, 2003).

Seminario (2004) refiere que en Perú, la superficie cultivada varía entre 2000 y 4500 ha/año, donde el Departamento de Cajamarca representa el 51-80\%, con rendimientos que van de 5000 a $6000 \mathrm{~kg} / \mathrm{ha}$. Bueno (2004) citado por Heredia et al. (2009) indica que en Brasil la arracacha se cultiva, principalmente, en las regiones del sur y sureste, en pequeñas áreas, con poco uso de insumos. El área de plantación es de aproximadamente 16.000 ha, siendo Paraná y Minas Gerais, los principales estados productores.

A pesar de su alta potencialidad para la agroindustria y sus buenas cualidades nutricionales (Hermann, 1997; Jiménez, 2005; Rodríguez et al. 2004) es una especie a la que no se le ha dado suficiente importancia dentro de los planes de desarrollo agrícola, tanto nacionales, como departamentales.

En el municipio de Boyacá, su explotación se concentra en manos de pequeños productores, desarrollando, por décadas, tecnologías de cultivo propias. Algunas veces estas prácticas no son las más adecuadas, pero la conversión hacia una alta tecnificación puede no ser la 
mejor alternativa. En la actualidad, la producción agrícola es la principal fuente de ingresos de los productores, quienes cultivan otras especies, como papa, arracacha, maíz, arveja, frutales caducifolios (manzana, durazno, ciruelo), curuba, mora, tomate árbol, entre otros; debido al microfundio existente, la producción es diversificada y asociada, con las siguientes combinaciones: papaarveja, haba-maíz y arveja, maíz-frijol, arracacha-maíz; la ganadería ocupa el $21 \%$ del área y la comercialización de los productos, se realiza, principalmente, los domingos en el municipio y en las localidades cercanas, como Ramiriquí, Jenesano, Tunja y Bogotá (POT Municipal, 2003).

Considerando lo expuesto, los objetivos del presente estudio fueron conocer las tecnologías y las prácticas de manejo de la arracacha a nivel local, teniendo como base la documentación de las experiencias de sus productores y generar un diagnóstico, fundamento para el planteamiento de estrategias de mejoramiento de la productividad y de la reducción de los costos de producción.

\section{MATERIALES Y MÉTODOS}

El presente estudio, se realizó en el municipio de Boyacá, ubicado en la Provincia de Márquez, centro-occidente del Departamento de Boyacá, a 05²7'15.1" de latitud norte y $073^{\circ} 21^{\prime} 48.6^{\prime \prime}$ de longitud oeste. Su temperatura promedio es de $14^{\circ} \mathrm{C}$ y la altitud oscila entre 2200 y 2900 msnm.

Con el objeto de conocer las experiencias de los productores y las tecnologías de producción, se efectuaron diez visitas de campo, entre diciembre de 2008 y junio de 2009, en las que se reconocieron 36 cultivos en las veredas Pachaquirá (9), Rupaguata (6), Soconzaque Occidente (5), Huerta Grande (5), Vanegas Sur (4), Rique (3), Centro (2) y Huerta Chica (2); se entrevistaron a los propietarios o las personas que conocían el manejo de los cultivos. La encuesta aplicada, se formuló con preguntas abiertas sobre cada uno de los aspectos a evaluar. La unidad de muestreo consistió en una finca, donde se encontraba establecido un cultivo de arracacha. No se halló información que permitiera definir el tamaño y las características de la población objetivo, por tanto, se definieron en el transcurso del trabajo de campo.
Con las visitas y la aplicación de encuestas, se elaboró un diagnóstico participativo, que valorara los conocimientos de los productores y permitiera conocer la problemática que los aqueja. La información recopilada, mediante la encuesta, estuvo enfocada en el sistema de siembra, realización de prácticas culturales, como deshierbas y aporque, fertilización, manejo fitosanitario, materiales genéticos cultivados y asistencia técnica. Debido a su complejidad, no se presentan resultados sobre los costos de producción del cultivo.

\section{RESULTADOS Y DISCUSIÓN}

Los productores manifiestan que la evolución del sistema de producción de arracacha, también conocida como coya en el municipio de Boyacá, se ha dado desde una perspectiva de agricultores minifundistas, que la cultivaban como producto de pan coger, hacia una explotación de mayores áreas con fines comerciales.

Según los productores, las veredas con mayor área cultivada en arracacha son Rupaguata, Pachaquirá y Rique, aunque en este estudio no se realizó una evaluación cuantitativa al respecto. La mayoría de ellos, la han cultivado toda su vida, pues es una tradición adquirida de sus padres y, a su vez, de ancestros. Cerca del $30 \%$ de los encuestados, que corresponde a los productores jóvenes, de edad entre 25 y 40 años, indican que llevan menos de cinco años en la explotación comercial de esta especie.

El área de las fincas visitadas variaba entre 0,5 y 20ha, la mayoría (70\%, aproximadamente) con menos de 5ha. El 50\% de los cultivos de arracacha tienen menos de 1 ha y el $22 \%$ entre 1 y 3 ha, es decir, se consideran minifundios.

El rango de altitudes en el que se ubican los cultivos visitados oscilan entre de 2200 a $2600 \mathrm{msnm}$. Los productores indican que cuanto más alto se encuentra el lote de siembra, más tarda la cosecha. Uno de los productores manifestó que la variedad yema de huevo, sembrada en sitios a más de $2600 \mathrm{~m}$ de altitud, tiene un ciclo dos meses más largo (11 meses) respecto a plantaciones ubicadas en altitudes menores a 2300 m (9 meses). Aunque no especifica la duración del periodo vegetativo, Higuita (1968) reporta observaciones similares al comparar cultivos ubicados en el municipio de La Ceja (Antioquia), a 2240msnm y la Sabana de 
Bogotá, a 2640msnm, hallando que este último es más largo. En estudios del INIAP $(1988,1989)$ en la estación Santa Catalina (3050 msnm) en Ecuador, se observó que por encima de $2800 \mathrm{~m}$ de altitud, la cosecha se presenta muy tardía, llegando hasta 370 días.

Sistema de plantío. El estudio mostró que 25\% de los productores entrevistados plantan la arracacha como monocultivo y $75 \%$ la asocian a especies anuales y/o perennes. Las semestrales y anuales son maíz y leguminosas, como fríjol, arveja, garbanzo y haba y las perennes son frutales, como el tomate de árbol, principalmente. Es muy importante el hecho que la arracacha sea cultivada en asociación, ya que su ciclo vegetativo es largo y, de esta manera, se optimiza el uso de la tierra, mejorando la economía familiar y la alimentación.

Se encontró que en la siembra se manejan dos sistemas: el tradicional por surcos y el 'mojoniado'. En este último, cada planta es instalada en una especie de montículo, independiente de las otras. Este sistema es ahora poco empleado, sin embargo, fue habitual hace algunas décadas. La distancia de plantío más frecuente entre surcos fue de $80 \mathrm{~cm}$, en $47 \%$ de los cultivos visitados, seguida en menor proporción (31\%) por distancias entre 40 y $70 \mathrm{~cm}$ y otras (22\%). La distancia entre plantas, en la mayoría de casos (64\%), fue de 40 y $50 \mathrm{~cm}$ o de 20 a $30 \mathrm{~cm}$ (14\%). Bajo la variabilidad y las posibles combinaciones de distancias encontradas, la densidad de plantío fluctuó entre 21000 y 41000 plantas/ha. Jaramillo (1984) reporta que en Antioquia los cultivos son realizados con distancias de siembra de $1 \mathrm{~m}$ entre surcos y 0,5m entre plantas, para una densidad de 20000 plantas/ha. De igual forma, Vásquez E Gutiérrez (2000) señalan que para el municipio de Cajamarca (Tolima) estas mismas distancias son óptimas.

No se encontraron épocas del año definidas para realizar el plantío. En todos los meses, se reportaron siembras, lo que indica que no están sometidas a la estacionalidad de las lluvias. Según Higuita (1968), con una precipitación anual de $600 \mathrm{~mm}$ se pueden establecer cultivos sin necesidad de riego suplementario; por su parte, Jaramillo (1984) reporta requerimientos mínimos de $1000 \mathrm{~mm}$. En Perú, Amaya E Julca (2006) señalan que la arracacha es una planta rústica que se puede propagar durante todo el año, aunque la época de inicio de lluvias es más apropiada. Los factores más determinantes sobre el momento de realizar la plantación son la disponibilidad de material propagativo y de terreno, ya que la arracacha se usa como cultivo de rotación. Usualmente, los productores hacen rotaciones de esta raíz con maíz, papa y leguminosas, como fríjol, arveja y garbanzo o dejan 'descansar' el terreno con pastos.

Además de la arracacha, $64 \%$ de los encuestados cultiva otras especies, generalmente frutales, como tomate de árbol, uchuva, curuba y caducifolios, leguminosas (arveja, fríjol y garbanzo), maíz y papa. Es importante mencionar que en dos de las fincas visitadas se encontraron dos tipos, denominados localmente alverjuelo y lentejuelo, que según los agricultores se han dejado de plantar y tuvieron alguna importancia agrícola y alimentaria para sus antecesores.

Material de propagación. El material usado para propagación en campo se denomina localmente colino, que Hermann (1997) refiere como un tallo aéreo compuesto por internudos, nudos y cicatrices dejadas por las hojas que lo recubren. Los colinos, se cortan de plantas sanas alrededor de dos meses antes de la cosecha y se empacan en costales. Los productores refieren que al momento de cortarlos, se deben dejar con la menor cantidad de cepa posible, ya que este material origina, exactamente con estas características, plantas con mayor rendimiento de raíces comerciales. Un trabajo adelantado en Brasil, evaluando el plantío de arracacha con colinos grandes $(22,1 \mathrm{~g})$, medianos $(14,1 \mathrm{~g})$, pequeños $(9,7 \mathrm{~g})$ y muy pequeños $(5,7 \mathrm{~g})$, demostró que estos últimos presentan mejor rendimiento de raíces comerciales (Heredia et al. 2009). La evaluación del peso del colino, en seis cultivos del municipio de Boyacá, permitió establecer como promedio $35 \mathrm{~g}$, máximo $51 \mathrm{~g}$ y mínimo 21g.

Para la propagación, $61 \%$ de los productores utilizan colinos de sus propios cultivos, es decir, de la misma finca; $25 \%$, los consiguen en la misma vereda, mientras que $14 \%$, los adquieren en otra vereda.

Materiales genéticos. No se referirá aquí el concepto de variedad, ya que en la arracacha no se tiene claridad sobre este aspecto. Diferentes autores mencionan conceptos como cultivar, forma hortícola y accesión (Sánchez E Vásquez, 1996; Higuita, 1968; Jaramillo, 1984; Vásquez et al. 2004). Se usa el término 'material genético', que aquí hace referencia a un grupo de plantas 
que a simple vista se pueden agrupar de acuerdo con rasgos de color y de forma de sus estructuras y basado en sus características agronómicas.

De acuerdo con los conocimientos locales, se determinó la existencia de siete materiales, cuyos nombres comunes son: paliverde, palirrusia o palimorada, palinegra, yema de huevo o cartagenera, blanca de tarro, amarilla de tarro y yucatana. Estos materiales se diferenciaron, principalmente, por el color de las hojas, de los tallos y de las raíces, como se explica en la tabla 1. Estas mismas variables fueron utilizadas por Vásquez et al. (2004) en la caracterización morfológica de la colección colombiana de arracacha. Para el caso de la raíz, se encontró que podía ser blanca o amarilla, en algunos materiales, con presencia de un anillo de color morado. Seminario (2004) menciona que existen cuatro tipos de arracacha: de pulpa blanca, de pulpa amarilla, de pulpa blanca con pigmentación púrpura y de pulpa amarilla con pigmentación púrpura. Higuita señala que en 1968, en el C.I. ICA “Tibaitatá” (Mosquera, Cundinamarca), se tenían nueve colecciones de material vegetal de arracacha y que las bases para diferenciarlas eran el color del follaje, de la raíz y del anillo presente en la raíz. En la zona de estudio, se encontraron hojas de color verde con diferente intensidad, verde-morado o morado intenso y raíces de color blanco y amarillo, con o sin presencia de un anillo de color morado.

De acuerdo con la forma y la estructura de la raíz, los agricultores definen dos clases de materiales: una de las clases agrupa los materiales cuya raíz principal es gruesa, que denominan "tarro", con raíces secundarias pequeñas, conocidas como apios, dentro de la cual, se encuentran los materiales yucatana, blanca de tarro y amarilla de tarro. Esta clase es conocida vulgarmente como "variedades de tarro". En la otra, se reúnen los materiales con raíz central más pequeña que la de la primera clase, con grandes y numerosos apios, donde agrupan los materiales paliverde, palirrusia, palinegra y yema de huevo, conocidas como "variedades de apio". Hacia los 50's, se cultivaban solamente los materiales blanca de tarro, amarilla de tarro y palinegra. Posteriormente, se efectuaron introducciones desde Cajamarca (Tolima), de los materiales denominados palirrusia y paliverde, por agricultores que trabajaron allí y regresaron al municipio de Boyacá, trayendo material propagativo. Hace diez años o menos, se introdujo la variedad yema de huevo, también desde Cajamarca.

Tabla 1. Color característico de los principales órganos de las plantas de arracacha, en cada uno de los materiales genéticos encontrados en el municipio de Boyacá.

\begin{tabular}{|c|c|c|c|c|}
\hline Nombre común & $\begin{array}{c}\text { Color de } \\
\text { la hoja }\end{array}$ & Color del tallo & $\begin{array}{c}\text { Color predominante } \\
\text { en la raíz }\end{array}$ & $\begin{array}{c}\text { Presencia de anillo } \\
\text { morado en la raíz }\end{array}$ \\
\hline Amarilla de tarro & Verde & Verde & Amarillo & No \\
\hline Blanca de tarro & Verde & Verde & Blanco & Sí \\
\hline Paliverde & Verde & Verde & Amarillo & No \\
\hline Palirrusia & Verde & Morado suave & Amarillo & No \\
\hline Palinegra & Verde- Morado & Morado & Amarillo & No \\
\hline Yema de huevo & Verde & Verde & Amarillo & Sí \\
\hline Yucatana & Morado oscuro & Morado suave & Amarillo & \\
\hline
\end{tabular}

* Con el nombre blanca de tarro los productores identifican dos materiales, uno que presenta anillo morado en la raíz y otro que no lo presenta. 
Los materiales de tarro, en la actualidad, no son comerciales, por eso se ha disminuido su área de plantío hasta quedar confinada a pequeños lotes de productores que la mantienen para auto-consumo y para "no dejar perder la semilla”; esta situación preocupa por el riesgo de pérdida de la biodiversidad. El material paliverde es cultivado por su alta producción, aunque en los últimos años se ha impuesto el plantío de yema de huevo, dada su aceptación en el mercado.

De acuerdo con sus experiencias, los agricultores manifestaron que los materiales yema de huevo y blanca de tarro son más precoces, con 12 a 15 meses del plantío a la cosecha; palirrusia, de 12 a 18 meses, lo mismo que paliverde y palinegra, pero pueden llegar a ser cosechadas a los 24 meses, caso poco frecuente.

Suelos y preparación. La topografía de la región es quebrada. En los cultivos visitados predominan los suelos de texturas franco-arcillosas y arcillosas, seguidas por la arcillosa y arenosa. Jaramillo (1984) menciona que la arracacha requiere suelos profundos, friables y con alto contenido de materia orgánica; en la zona de estudio, no se observó que la textura fuera una limitante para la producción, a pesar que se señala que en suelos arcillosos a franco-arcillosos, el engrosamiento de las raíces es limitado.

La preparación del suelo para el plantío consiste, generalmente, en arar y rastrillar; el surcado se hace con arados de tiro jalonados por caballos y bueyes o con azadón. Cuando el terreno proviene de cultivo de papa, se encuentra en buenas condiciones y tan solo se ara con bueyes o se rastrilla. En Antioquia, Jaramillo (1984) dice que la preparación de los suelos es realizada de forma similar.

Riego. Un alto porcentaje de los productores $(86 \%)$ no dispone de un sistema de riego, el restante $14 \%$, cuenta con riego por aspersión o por gravedad. Lo anterior, se debe a que la mayoría no dispone de fuentes de agua $\mathrm{y}$, aunque no se han determinado los requerimientos hídricos de la especie, las experiencias en campo indican que pueden ser suplidos por precipitaciones entre 600 y 1000mm/año (Higuita, 1968; Jaramillo, 1984). En el municipio de Boyacá, la precipitación promedio anual es de $911 \mathrm{~mm}$ (POT Municipal, 2003). En el marco del presente estudio, no se realizó evaluación sobre las diferencias de productividad entre cultivos con o sin riego.
Fertilización. Casi la totalidad de los entrevistados manifestaron que no realizan análisis de suelo, por tanto, la fertilización se planea de forma empírica. Situación similar es reportada por Vásquez E Gutiérrez (2000), en el municipio de Cajamarca.

En el municipio de Boyacá, $75 \%$ de los productores emplea únicamente abonos químicos; $11 \%$, aplica tanto abonos orgánicos como químicos y, 14\%, solamente abonos orgánicos. En el caso de los productos químicos, el más empleado es el abono compuesto $15-15-15$ y otras fuentes como 18-18-18, 10-30-10 y 13-26-6. Entre los orgánicos, se reportaron el estiércol, el pasto descompuesto, la gallinaza y el humus. De los productores encuestados, $36 \%$ abona una sola vez durante el ciclo de cultivo; $39 \%$ dos veces y $17 \%$, tres momentos. La distribución de estas aplicaciones está sujeta a las precipitaciones y a la realización de otras labores como la deshierba, ya que con frecuencia estas dos labores se efectúan al mismo tiempo.

No es posible determinar si las dosis y fuentes de nutrientes son adecuadas, debido a la falta de estudios sobre el comportamiento de los materiales genéticos a factores como clima y fertilización. El trabajo de Vásquez y Gutiérrez (2000), concluye que la respuesta de las variedades estudiadas amarilla paliverde y amarilla palirrusia, a diferentes fuentes de fertilización química (urea $46 \%$, fosfato diamónico, superfosfato triple, cloruro de potasio), no fue consistente. Para la Sabana de Bogotá, Jaramillo (1984) reporta que se han obtenido buenos rendimientos, con aplicaciones de 500 a $600 \mathrm{~kg} /$ ha de abono 10-30-10 más 3t/ha de gallinaza.

Enfermedades. La pudrición de raíz fue el problema fitosanitario más frecuente, mencionado por $72 \%$ de los entrevistados. Análisis de tejidos de la raíz realizados en el Centro de Diagnóstico en Sanidad Vegetal de la UPTC (Tunja) indicaron que el agente causal es el hongo Sclerotinia sclerotiorum, ampliamente reportado como patógeno de la arracacha (Hermann, 1997; Aguirre E Vásquez, 2000; Dos Santos, 2004). El síntoma inicial de su ataque es un marchitamiento del follaje y, posteriormente, al arrancar la planta su raíz presenta pudrición y estructuras del patógeno, llamadas esclerocios. Los agricultores desconocen el manejo de la enfermedad y por eso no realizan ningún tipo de control. Los demás productores hicieron referencia a otras enfermedades, que denominaron ceniza, 
Fusarium, enroscamiento de la hoja y arrebolado, pero se desconocen los agentes causales, ya que hasta la fecha no se ha realizado ningún estudio.

Plagas. No se reportaron daños de nivel económico, excepto los causados por babosas, no identificadas, cuando atacan al inicio del ciclo, época en que consumen las primeras hojas o la raíz, pudiendo causar la muerte y, por tanto, la pérdida de plantas. Se realiza control, mediante el encalado previo al plantío o la aplicación al suelo de gránulos, de algún producto comercial, a base de metaldehído.

Los agricultores también reportaron el daño de una larva conocida como centella, que consume el follaje. En el Laboratorio de Entomología de la UPTC (Tunja), se identificó como Papilio polyxenes asterius Stoll (Lepidoptera: Papilionidae); esta misma especie es reportada por Sánchez E Vásquez (1996), en Cajamarca y por Montaldo (1991), en Venezuela.

Indican la existencia de otras larvas, comedoras de follaje de menor importancia, que durante los recorridos no se observaron. Para el control emplean productos a base de ingredientes activos, como clorpirifos, metamidofos, malathion y profenofos, que son aplicados también a la papa.

Malezas. En los cultivos de arracacha, se localizaron diferentes especies de malezas. Dentro de las gramíneas, principalmente kikuyo (Kikuyuochloa clandestina (Hochst. ex Chiov.) H. Scholz y raigrás (Lolium perenne L.) y en el grupo de hoja ancha, la romasa (Rumex sp.) y el barbasco (Polygonum segetum Kunth). Los métodos de control, se fundamentan en el deshierbe manual, tres o cuatro veces en un ciclo de cultivo y la aplicación de herbicidas, en base a diuron y fluazifopp-butil. En Cajamarca, el control químico también se realiza con productos de diuron y, en forma manual, cuatro y ocho meses después de siembra (Vásquez $\mathcal{E}$ Gutiérrez, 2000).

Cosecha y rendimiento. Los productores indicaron que, dependiendo de los materiales genéticos, el momento de la cosecha variaba entre 12 y 18 meses, aunque algunos agricultores atrasaban la cosecha, incluso hasta los 24 meses. Se observó que la demora en la cosecha se debía a la espera del productor por mejores precios en el mercado, ya que en determinadas épocas éste se satura. Por otra parte, la disponibilidad de mano de obra y de transporte les hace optar por demorar unos meses más la cosecha o por realizar la cosecha escalonada, es decir, recolectar y vender pequeñas cantidades periódicamente, hasta cosechar la totalidad del lote.

Quienes están acostumbrados a cosechar tardíamente manifiestan que la calidad de la raíz no disminuye; sin embargo, otros productores consideran que existe mucho riesgo por el ataque de enfermedades y la pérdida de calidad de la raíz que, se torna fibrosa y cambia de sabor.

Ninguno de los productores entrevistados ha calculado el rendimiento obtenido en sus cultivos, por tanto, aquí se reportan datos aproximados. De acuerdo con el $67 \%$ de los encuestados, al momento de la cosecha el rendimiento oscila entre 2 y $3 \mathrm{~kg}$ por planta (peso de raíz); el $14 \%$ indicaba que variaba entre 4 y $5 \mathrm{~kg} /$ planta y los productores restantes (29\%) desconocen por completo los rendimientos promedio o potenciales. Debido a este desconocimiento y a las grandes variaciones en la densidad de siembra (21.000 a 41.000plantas/ha, aproximadamente), no fue posible hacer un estimativo de los rendimiento. El MADR (2008), reporta un rendimiento de $12 \mathrm{t} /$ ha en este municipio, siendo el promedio nacional de 11,9 t/ha.

Empaque y sitio de venta. Para su comercialización la arracacha se empaca en costal de fibra blanco, de $62,5 \mathrm{~kg}$. De los encuestados, $92 \%$ citaron que venden sus cosechas en las ciudades de Tunja y/o Ramiriquí y, los $8 \%$ restantes, en la plaza de mercado de Boyacá o en Bogotá y a intermediarios que la adquieren en el mismo lote. Las cosechas más grandes son llevadas a Tunja, Bogotá y/o Ramiriquí; localmente, en la plaza de Boyacá, se comercializan cantidades más pequeñas. Acerca del uso y destino del producto, 39\% indicó que produce solo para la venta y $53 \%$ tanto para la venta como para auto-consumo.

Asistencia técnica. Ninguno de los encuestados en las veredas productoras de arracacha indicó que ha recibido asistencia técnica para el cultivo de esta especie. Esta situación, se relaciona con la imposibilidad económica de sus cultivadores para sufragar los costos de un asistente técnico y la falta de profesionales con amplios conocimientos sobre esta especie. Se suma a lo anterior, 
la falta de costumbre para solicitar asistencia técnica en los cultivos tradicionales cuyas técnicas de producción son mejor conocidas.

En la zona de estudio no existen asociaciones de productores de arracacha; no obstante, la mayoría manifestaron el deseo de asociarse para tener acceso a capacitación, asistencia técnica y mejoras en la comercialización.

En explotaciones pequeñas, la mano de obra, para muchas de las labores, es de tipo familiar. En áreas mayores, se contrata por jornal o por labor, aunque se sigue haciendo uso de la mano de obra familiar.

Como consideraciones finales del trabajo y dada la importancia del sistema productivo de la arracacha, no solo en el municipio de Boyacá, sino en varios municipios del Departamento, se detectó la necesidad de una mayor atención por parte de las entidades encargadas de diseñar y de ejecutar las políticas de desarrollo agrícola.

A pesar de observarse que la tecnificación del cultivo es baja, cálculos basados en los datos de rendimiento, suministrados por los productores, permiten afirmar que el municipio se encuentra entre los de mayor productividad a nivel nacional, por lo cual, se recomienda plantear alternativas de manejo agroecológico, encaminadas a mantener una baja incidencia de plagas y enfermedades.

Financiación. El presente estudio, se realizó en el marco del Convenio 104-08 entre la Corporación PBA y la UPTC, con recursos del Ministerio de Relaciones Exteriores de Holanda. Conflicto de intereses: Esta investigación y el manuscrito fueron realizados por todos los autores, por tanto, declaramos que no existe ningún conflicto de intereses que ponga en riesgo la validez de la presente publicación.

\section{BIBLIOGRAFÍA}

1. AGUIRRE, M.; VÁSQUEZ, M. 2000. Evaluación de plantas de arracacha (Arracacia xanthorrhiza Bancroft) provenientes de semilla sana y afectada por "argeniado". Rev. ICA Nataima (Colombia). 5:65-72.
2. AMAYA, J.; JULCA, J. 2006. Arracacha, Arracacia xanthorrhiza Bancroft. Gerencia Regional de Recursos Naturales y Conservación del Medio Ambiente. Gobierno Regional La Libertad. Perú. 15p.

3. DOS SANTOS, F. 2004. Producción de arracacha en Brasil. En: Seminario, J. (ed.). Raíces Andinas: Contribuciones al conocimiento y a la capacitación. Serie: Conservación y uso de la biodiversidad de raíces y tubérculos andinos: Una década de investigación para el desarrollo (1993-2003) No. 6. Universidad Nacional de Cajamarca, Centro Internacional de la Papa, Agencia Suiza para el Desarrollo y la Cooperación. Lima, Perú. p.285287.

4. HIGUITA, F. 1968. El cultivo de la arracacha en la Sabana de Bogotá. Separata Agricultura Tropical (Colombia). 14(3):139-147.

5. HEREDIA, N.; VIEIRA, M.; DIMAS, J.; GONZÁLEZ, P.; BRANDÃO, N.; MAZARON, B. 2009. Produtividade de mandioquinha-salsa sob diferentes densidades de plantio e tamanho das mudas. Ciência e Agrotecnologia (Brasil). 33(1):139-143.

6. HERMANN, M. 1997. Arracacia xanthorrhiza Bancroft. En: Herman, M.; Hiller, J. eds. Andean Roots and tubers: Ahipa, arracacha, maca and yacon. International Potato Center CIP. Lima Perú. p.75-172.

7. Instituto Nacional de Investigaciones Agropecuarias INIAP. 1988. Caracterización de 71 entradas de zanahoria blanca (Arracacia xanthorrhiza), en Santa Catalina. Programa de Cultivos Andinos Ecuador. Informe técnico anual. p.16-17.

8. Instituto Nacional de Investigaciones Agropecuarias INIAP. 1989. Evaluación y selección de 10 clones promisorios de zanahoria blanca en Santa Catalina. Programa de Cultivos Andinos Ecuador. Informe técnico anual. p.42-43.

9. JARAMILLO, J. 1984. El cultivo de la arracacha (Arracacia xanthorrhiza). ICA Regional 4. Curso de actualización en tecnología agrícola. Distrito de Rionegro. Documento de trabajo No. 14. p.179-189. 
10. JIMÉNEZ, F. 2005. Características nutricionales de la arracacha (Arracacia xanthorrhiza) y sus perspectivas en la alimentación. Publicación Virtual Red Peruana de Alimentación y Nutrición. Lima, Perú. 22p. Disponible desde Internet en: http:// www.rpan.org/monografias/monografia002.pdf (con acceso 12/05/09).

11. KNUDSEN, S.; HERMANN, M.; DOS SANTOS, F.; SORENSEN, M. 2004. Inducción de floración en el cultivo de arracacha (Arracacia xanthorrhiza Bancroft). En: Seminario, J. (ed.). Raíces Andinas: Contribuciones al conocimiento y a la capacitación. Serie: Conservación y uso de la biodiversidad de raíces y tubérculos andinos: Una década de investigación para el desarrollo (1993-2003) No. 6. Universidad Nacional de Cajamarca, Centro Internacional de la Papa, Agencia Suiza para el Desarrollo y la Cooperación. Lima, Perú. p.197213.

12. KNUDSEN, S.; ØRTING, B.; SØRENSEN, M. 2006. Multiplicación y conservación de arracacha (Arracacia xanthorrhiza Bancr.) y ajipa (Pachyrhizus ahipa (Wedd.) Parodi). En: Moraes, M; B. Øllgaard, B; Kvist, P; Borchsenius, F; Balslev H. (ed.) Botánica Económica de los Andes Centrales. Universidad Mayor de San Andrés, La Paz, Bolivia. p.483-508.

\section{MINISTERIO DE AGRICULTURA Y DESARROLLO} RURAL MADR. Secretarías de Agricultura Departamentales, Umatas. 2008. Arracacha: superficie cosechada, producción y rendimiento obtenido por departamento, años agrícolas 1997 - 2008. Disponible desde Internet en: www.dnp.gov.co/ PortalWeb/LinkClick.aspx?fileticket...tabid $=437$ (con acceso 08/08/09).

14. MONTALDO, A. 1991. Cultivo de raíces y tubérculos tropicales. $2^{a}$ Edición. Editorial Agroamérica. San José de Costa Rica. Instituto Interamericano de Cooperación para la Agricultura. 408p.
15. Plan de Ordenamiento Territorial del municipio de Boyacá. POT. 2003. Alcaldía Municipal de Boyacá, Departamento de Boyacá. 524p.

16. SEMINARIO, J. 2004. Origen de las Raíces Andinas. En: Seminario, J. (ed.). Raíces Andinas: Contribuciones al conocimiento y a la capacitación. Serie: Conservación y uso de la biodiversidad de raíces y tubérculos andinos: Una década de investigación para el desarrollo (1993-2003) No. 6. Universidad Nacional de Cajamarca, Centro Internacional de la Papa, Agencia Suiza para el Desarrollo y la Cooperación. Lima, Perú. p.1-38.

17. RODRÍGUEZ, G.; GARCÍA, H.; CAMACHO, J.; ARIAS, F.; RIVERA, J.; TORRES, F. 2004. La Harina de Arracacha. Manual técnico para su elaboración. Informe de investigación del Programa Nacional de Procesos Agroindustriales, CORPOICA. 24p.

18. SÁNCHEZ, G.; VÁSQUIEZ, N. 1996. Manejo de plagas en el sistema de producción de arracacha en el departamento del Tolima. Produmedios. Corpoica Regional 6. p.6-17.

19. VÁSQUEZ, N.; MEDINA, C.; LOBO, M. 2004. Caracterización morfológica de la colección colombiana (Tolima, Huila, Boyacá, Cauca) de arracacha (Arracacia xanthorrhiza). En: Seminario, J. (ed.). Raíces Andinas: Contribuciones al conocimiento y a la capacitación. Serie: Conservación y uso de la biodiversidad de raíces y tubérculos andinos: Una década de investigación para el desarrollo (1993-2003) No. 6. Universidad Nacional de Cajamarca, Centro Internacional de la Papa, Agencia Suiza para el Desarrollo y la Cooperación. Lima, Perú. p.165-178.

20. VÁSQUEZ, N.; GUTIÉRREZ, D. 2000. Fertilización y distancias de siembra en el cultivo de arracacha en el municipio de Cajamarca, Tolima. Revista ICA Nataima (Colombia). 5:57-64.

Recibido: Septiembre 3 de 2009

Aceptado: Mayo 3 de 2010 